\title{
Aplicação de métodos de biofísica no estudo da eficácia de produtos dermocosméticos
}

\author{
Gisele Mara Silva Gonçalves ${ }^{1 *}$, Patrícia Maria Berardo Gonçalves Maia Campos² \\ ${ }^{1}$ Faculdade de Ciências Farmacêuticas, Pontificia Universidade Católica de Campinas, ${ }^{2}$ Faculdade de Ciências \\ Farmacêuticas de Ribeirão Preto, Universidade de São Paulo
}

\begin{abstract}
O trabalho aborda a aplicação dos métodos de biofísica no estudo de eficácia de produtos dermocosméticos. A qualidade de um produto cosmético engloba a segurança de seu uso, a estabilidade da formulação, o aspecto, o sensorial e a sua eficácia. As técnicas de biofísica têm sido amplamente empregadas na avaliação do efeito de formulações dermocosméticas, principalmente devido ao fato de possibilitarem a avaliação de produtos em suas reais condições de uso, ou seja, na pele humana. A comprovação dos efeitos de produtos cosméticos e a elucidação de seu mecanismo de ação por meio de protocolos experimentais devidamente elaborados, que utilizem as técnicas de biofísica da pele, dentre outras, tornam-se de fundamental importância e, assim, a serem amplamente exploradas e ainda bastante desenvolvidas, visando à melhoria da qualidade de vida dos usuários de tais produtos.
\end{abstract}

Unitermos: Produtos dermocosméticos/controle de qualidade. Pele/biofísica/estudo. Biofísica/técnicas/ aplicação em cosmetologia. Cosméticos/eficácia.

\begin{abstract}
The authors consider the application of biophysical techniques in the study of cosmetics effectiveness. The quality of a cosmetic product includes the safety of its use, the formulation stability, the visual and other sensorial aspects, and its effectiveness. The biophysical techniques have been widely used in the evaluation of the effect of formulations of topical use, mainly due to the fact of facilitating the evaluation of products in its real use conditions, that is to say, directly in the human skin. The confirmation of the effects of cosmetic products and its action mechanism, by means of experimental protocols properly elaborated using the skin biophysics is of fundamental importance. It should be thoroughly explored and quite developed seeking the improvement of the life quality of users of such products.
\end{abstract}

Uniterms: Dermocosmetic products/quality control. Skin/biophysics/study. Biophysics/techniques/ cosmetologic application. Cosmetics/effectiveness.

\section{INTRODUÇÃO}

O perfil de qualidade de um produto cosmético engloba eficácia e segurança de seu uso, a estabilidade da formulação e o aspecto sensorial. As técnicas de Biofísica da pele têm sido amplamente empregadas na avaliação do efeito de formulações de uso tópico, principalmente por possibilitar a avaliação de produtos em suas reais condições de uso, ou seja, diretamente na pele humana (Hadgraft, 2001; Dujardim et al., 2002; Reuther et al., 2007).

\footnotetext{
* Correspondência: G. M. S. Gonçalves. Faculdade de Ciências Farmacêuticas, Pontifícia Universidade Católica de Campinas, Av. John Boyd Dunlop, s/n, - Jardim Ipaurussurama - 13059-900 - Campinas - SP, Brazil. E-mail: gmsg@puc-campinas.edu.br.
}

A comprovação da eficácia destes produtos é de suma importância para o mercado consumidor, que deseja ver concretizado o apelo de venda que o impulsiona para o ato da compra. Desta maneira, novas metodologias de avaliação desta categoria de produto têm sido continuamente desenvolvidas, com ênfase na comprovação científica dos reais benefícios propostos por estas formulações.

A constante pesquisa do tecido cutâneo e do emprego de metodologias não-invasivas tem dado origem a diferentes equipamentos para a avaliação dos efeitos dos produtos dermocosméticos (Dikes, 2002; Es-Spiekman, Lucassen, 2000; Smith et al., 2002; Oba et al., 2002; Egawa et al., 2002; Rodrigues, 1997; Zhai et al., 2008, Chilcott, Farrar, 2008, Campos et al., 2008; Dujardin et al., 2002). 
As técnicas de biofísica da pele são um dos mais importantes avanços tecnológicos na área da pesquisa dermatológica e tem tido repercussão nas demais áreas direta ou indiretamente a ela relacionadas. Estas técnicas consistem no estudo das características biológicas, mecânicas e funcionais da pele, por meio da medição de determinadas variáveis, por métodos não-invasivos cientificamente comprovados (Wiechers, Barlow, 2000; Pierard et al., 1995; Egawa et al., 2002; Lomuto et al., 1995; Henry et al., 1996; Oba et al., 2002; Silver et al., 2003; Le Fur, 2002; Berardesca, 1994; Benaiges et al., 1998).

Existe grande número de variáveis que podem ser consideradas como indicativas do estado em que se encontra a pele em relação à sua integridade e qualidade, em especial da epiderme, que podem ser mensuradas com simplicidade de operação, por meio da Biofísica da pele (Reuther et al., 2007). Exemplos claros são as análises da umectação cutânea, do teor de gordura (ou sebo), do valor de $\mathrm{pH}$ da superfície cutânea, da medida da perda transepidérmica de água e avaliação do relevo cutâneo (Egawa et al., 2002; Lodén, 2006; Hashimoto-Kumasaka et al., 1993). Além disso, é ainda possível monitorar as condições das camadas mais profundas, como a derme, por meio da determinação das propriedades mecânicas da pele (Zhai et al., 2008, Chilcott, Farrar, 2008; Ambroisine et al., 2007).

O potencial de aplicação dessa tecnologia tornou-se imenso, sendo possível abordar aspectos como a análise e caracterização do estado funcional da superfície da pele, a quantificação e caracterização de certas afecções cutâneas e análise dos efeitos terapêuticos ou cosméticos (Ambroisine et al., 2007; Berardesca, Maibach, 2007). Além disso, no Brasil, quando se atribui um benefício específico a um produto, deve haver comprovação para efetuar o pedido de registro no Ministério da Saúde (Guia, 2006).

A investigação é realizada na pele de voluntários e nas condições de uso desses produtos, sendo de grande valia para a elucidação científica dos benefícios atribuídos a estas formulações, como, por exemplo, o aumento da umectação e atuação na viscoelasticidade cutânea (Campos et al., 2008; Lodén, 2006; Walker, 1996; Rogiers, 1999). Como esses estudos permitem realizar análises quali e quantitativas, evita-se o erro de uma apreciação apenas subjetiva (Rodrigues, 1997). Em outras palavras, os estudos que antigamente eram realizados apenas a partir de informações clínicas e subjetivas podem ser enriquecidos pelo emprego dos métodos de biofísica da pele.

No Brasil, o Conselho Nacional de Saúde regulamentou as pesquisas envolvendo seres humanos através da Resolução 196/96 e constituiu a Comissão Nacional de
Ética em Pesquisa (CONEP), responsável, entre outros, pelo registro dos Comitês de Ética em Pesquisa institucionais. Todos os projetos de pesquisa envolvendo seres humanos devem obedecer às recomendações dessa Resolução. Assim, antes da avaliação de produtos cosméticos em humanos, todo e qualquer protocolo de estudo deve ser submetido à avaliação prévia por um Comitê de Ética.

Cumpre salientar que as condições de estudo e o protocolo de trabalho empregado nas medidas não-invasivas necessitam ser validados para a obtenção de dados confiáveis e condizentes com a realidade. Conforme será mencionado ao longo deste texto, as condições ambientais exercem influência sobre algumas variáveis, necessitando de uma padronização (Rohr, Schrader; 1998). De maneira geral, os voluntários são selecionados de acordo com o seu tipo de pele, sexo, idade e hábitos específicos. Dependendo do tipo de produto avaliado e do seu efeito esperado, são selecionadas diferentes regiões corporais, desde a face, antebraços e, até mesmo, a região dos tornozelos (Bazin, Fanchon, 2006; Chilcott, Farrar, 2008).

Neste breve artigo, serão abordados os métodos de biofísica utilizados para a obtenção das medidas do conteúdo aquoso do estrato córneo, da perda transepidérmica de água e das propriedades mecânicas da pele, por serem muito empregados, atualmente, na avaliação dos efeitos de formulações para os cuidados da pele, principalmente aquelas com apelo antienvelhecimento.

\section{Determinação do conteúdo aquoso do estrato córneo e da perda transepidérmica de água}

O filme hidrolipídico presente na superfície da pele, constituído por material graxo excretado pelas glândulas sebáceas e componentes excretados no suor, forma a chamada emulsão epicutânea. Esta emulsão possui diversas funções, sendo que protege a pele contra o ressecamento, participa na manutenção de sua flexibilidade e na formação da barreira de proteção acídica que evita a penetração de agentes externos prejudiciais ao organismo (Smith, 1999; Spencer, 1988). O conteúdo aquoso do estrato córneo refere-se a este filme, sendo que pode ser influenciado pela fisiologia cutânea e pelo ambiente (Chilcott, Farrar, 2008; Verdier-Sevrain, Bonté, 2007).

$\mathrm{O}$ equilíbrio adequado entre os componentes do filme hidrolipídico é fundamental para evitar a irritação cutânea e até mesmo algumas doenças. Como exemplo da alteração deste equilíbrio, pode ser citada a aplicação de agentes tensoativos na pele, que removem parte dos lipídios. Entretanto, na pele saudável isso não é alarmante, pois as glândulas sebáceas atuam imediatamente e, dentro de duas horas, em média, o filme hidrolipídico deverá 
estar complemente restabelecido (Tsai, Maibach, 2000; Verdier-Sevrain, Bonté, 2007).

Outro fator fundamental para o balanço de hidratação da pele é a integridade do estrato córneo. O conteúdo aquoso do estrato córneo é um dos mais importantes parâmetros na avaliação da função barreira, além da chamada perda transepidérmica de água. Quando a pele está adequadamente hidratada, ela está apta para, assim, cumprir efetivamente todas as suas funções. $\mathrm{O}$ aumento excessivo da perda transepidérmica de água indica que a função barreira da pele está prejudicada, ou seja, a pele está susceptível a agentes externos e, também, à desidratação (Verdier-Sevrain, Bonté, 2007; Madison, 2003; Kalia et al., 1998).

O filme hidrolipídico pode ser influenciado fisiologicamente pelo mecanismo de controle da temperatura corporal e pelo ciclo circadiano. Este ciclo, regulado pela intensidade da luz ao qual o corpo fica exposto, refere-se a todas as modificações ocorridas no metabolismo pelo período de 24 horas, sendo que podem ocorrer diferenças na temperatura corporal e, também, na perda transepidérmica de água (Le Fur, 2002).

Em relação à influência ambiental, a incidência de luz direta pode promover o aquecimento da pele e do ambiente, causando aumento da transpiração, pois, de acordo com Rohr \& Schrader (1998), que estudaram a influência de variações climáticas sobre a umectação cutânea, a estação do ano e o clima influenciam esta variável. Em situações de baixa umidade relativa do ar, o conteúdo de água do filme hidrolipídico tende a diminuir (Verdier-Sevrain, Bonté, 2007). Por outro lado, quando a temperatura ambiental e a umidade do ar são altas, a transpiração aumenta, porém, menos água pode evaporar da pele, tornando o estrato córneo umectado. Outros fatores que podem influenciar as condições de umectação cutânea são: o estilo de vida da pessoa, sua alimentação, o consumo regular de nicotina e álcool, estresse e o uso de produtos farmacêuticos e cosméticos (Leow, Maibach, 1998; Fukuda et al., 2001a,b).

Dentre os diversos métodos disponíveis para a medida do conteúdo aquoso do estrato córneo, os principais são: a Espectroscopia do Infravermelho, que é baseada na absorção dérmica de ondas no infravermelho e depende do conteúdo aquoso da pele; a Freqüência de Ressonância, baseada na medida da umectação pela transmissão de ondas, que depende da elasticidade da pele; a Ressonância Magnética Nuclear (NMR) baseada na densidade protônica; a Descamação, baseada no processo natural de descamação do estrato córneo; a Medida da Impedância, baseada na medida de sua resistência elétrica; e, finalmente, o Principio da Capacitância, o qual baseia-se nas diferenças entre a constante dielétrica da água e de outras substâncias. Porém, o método da capacitância apresenta a vantagem de não sofrer a interferência de sais ou produtos químicos aplicados sobre a pele e este tem sido o método de escolha na maioria dos trabalhos recentemente publicados, em termos de avaliação do efeito de formulações tópicas (Rohr, Schrader, 1998; Verdier-Sevrain, Bonté, 2007; Campos et al., 2008).

Em relação aos cosméticos, a umectação cutânea pode, na maioria das vezes, ser melhorada ou corrigida pelo uso destes produtos. O equilíbrio do conteúdo aquoso no estrato córneo é imprescindível para a manutenção da eudermia, ou seja, das condições de normalidade da pele, que é o objetivo das formulações cosméticas (Brasil, 2008). Assim, considera-se que a comprovação dos efeitos biológicos das formulações hidratantes é de grande interesse, pois manter a pele adequadamente umectada retarda o envelhecimento cutâneo e melhora o seu aspecto geral, bem como previne ou trata certas doenças de pele. Além disso, essa classe de produtos tem sido considerada dominante na área cosmética (Gaspar et al., 2001; Pinto et al., 1997; Baran, Maibach, 1998; Lodén, 2006; Tabata et al., 2000; Ambroisine et al., 2007).

A função de barreira da pele, que é o controle sobre a perda de líquidos que ocorre por transpiração e evaporação, é de fundamental importância, dentre todas as funções que a pele exerce no organismo. A pressão formada pela difusão da água pode ser medida como perda transepidérmica de água, expresso em gramas por $\mathrm{m}^{2}$ por hora. Portanto, a chamada perda transepidérmica de água é a perda total de água da epiderme viável e derme, por difusão através do estrato córneo para a superfície e originada das glândulas sudoríparas (Rossi, Vergnanini, 1997).

A medida da perda transepidérmica de água tem sido um dos parâmetros usados para a avaliação dos efeitos que o meio ambiente, a temperatura, algumas doenças e também formulações aplicadas topicamente exercem nas trocas de água da pele com o ambiente. Quando a integridade da pele está danificada, a perda transepidérmica de água tende a aumentar, ao passo que quanto mais perfeita for a cobertura de proteção da pele, ou barreira, maior será o conteúdo aquoso do estrato córneo. A pele saudável tende a apresentar baixos valores de perda transepidérmica de água e altos valores de conteúdo aquoso do estrato córneo (Hadgraft, 2001).

Os lipídios extracelulares que compõe o filme hidrolipídico exercem importante papel na função de barreira, e consistem de uma mistura de ceramidas, colesterol e ácidos graxos, adicionados de pequenas quantidades de sulfato de colesterol, glucosil ceramidas e fosfolipídeos (Verdier-Sevrain, Bonté, 2007; Petersen, 1992; Summers 
et al., 1996). A permeabilidade da pele a agentes irritantes depende do número de anexos cutâneos (folículos capilares e glândulas sudoríparas), da espessura da pele, do número de camadas celulares e do conteúdo lipídico do estrato córneo (Benson, 2005; Schuplein et al., 1993).

A integridade do estrato córneo é, provavelmente, o fator mais importante na resistência da pele a irritantes e sensibilizantes, e pode ser facilmente monitorada usando medidas da perda transepidérmica de água. Segundo alguns autores, a maioria dos casos de problemas na pele, como a tendência a apresentar erupções e irritação, pode estar relacionada ao comprometimento da função de barreira (Yokota et al., 2002; Haftek, 2002, Maes et al., 1990; Rieger, 1992). A irritação pode alterar os lipídios dos interstícios do estrato córneo, o que resulta, paralelamente, em aumento temporário na perda transepidérmica de água. De forma geral, essas alterações iniciam-se antes do aparecimento de quaisquer indicações visíveis da irritação, sendo a medida da perda transepidérmica de água um método útil, sob condições definidas, para se predizer a irritação causada por um produto (Maes et al., 1990).

Durante o envelhecimento não há uma correlação precisa entre a perda transepidérmica de água e o grau de modificações degenerativas, sendo que a mesma tende à redução. Esse decréscimo é particularmente evidente após os 60 anos e mais proeminente nas áreas expostas ao sol, onde a agressão actínica é um fator predominante de acentuação do envelhecimento. O decréscimo simultâneo da perda transepidérmica de água e do conteúdo aquoso do estrato córneo é uma das características da pele envelhecida. Assim, a pele envelhecida caracterizada popularmente como "pele seca" e que apresenta um aspecto opaco e com linhas mais marcadas na superfície, pode ser distinguida da pele que apresenta algum tipo de patologia que a deixa com aspecto semelhante, uma vez que, nesta última, a função de barreira foi modificada por outros fatores (Hadgraft, 2001; Milan et al., 2007; Berardesca, Borroni, 1995).

A fisiologia também exerce influência na perda transepidérmica de água, pois a pele realiza uma troca gasosa com o ambiente, que consiste da difusão de oxigênio e dióxido de carbono através de sua superfície. Esta troca representa apenas $1,5 \%$ da troca total de gases no organismo, que é de grande importância, sendo influenciada pela temperatura e pela umidade relativa do ar. O mecanismo de regulação da temperatura corporal também influencia a perda transepidérmica de água, pois o organismo compensa a diferença entre a temperatura corporal e a ambiente por meio da evaporação de água. Quanto maior a temperatura ambiente, maior a perda transepidérmica de água. Já a umidade relativa do ambiente não tem correlação direta com a perda transepidérmica de água, mas o seu aumento provoca queda na difusão passiva e nos valores de perda transepidérmica de água (Kemenade et al., 2004).

Em relação ao estudo dos efeitos de formulações cosméticas na pele, as medidas da perda transepidérmica de água são essenciais para a elucidação dos mecanismos de atuação de algumas formulações com finalidades de prevenir o envelhecimento cutâneo. Nem todos os produtos tidos como hidratantes atuam retendo a água na pele devido às características higroscópicas que apresentam, e sim prevenindo a sua perda através da criação de uma barreira superficial. Dessa maneira, a melhoria da pele é decorrente da combinação de agentes oclusivos e umectantes nestes produtos (Gonçalves, Maia Campos, 2004; Campos et al., 2008; Lodén, 2001; Rieger, 1998, Rieger, 1996; Rossi, Vergnanini, 1997; Baran, Maibach, 1998).

\section{Propriedades mecânicas da pele}

As propriedades mecânicas da pele têm sido objeto de estudos relacionados ao processo de cicatrização e formação de quelóides, bem como a avaliação de eficácia de produtos para os cuidados da pele (Smith et al., 2002; Oba et al., 2002; Serup, Northeved, 1985). Enfoque especial é dado às alterações que o envelhecimento provoca na pele, como a flacidez, o enrugamento, a desidratação e a perda de elasticidade cutânea. A importância da manutenção destas propriedades da pele provém da ação que esta exerce, por exemplo, quando exposta à traumas mecânicos, ou seja, fricção, impacto e pressão (Edwards, Marks, 1995; Diridollou et al., 1998; Fthenakis et al., 1991).

A pele possui uma estrutura complexa, devido à sua natureza multifuncional, e suas características gerais variam de acordo com o local do corpo. Em relação às propriedades mecânicas, a pele humana é viscoelástica, ou seja, apresenta tanto características elásticas quanto plásticas, devido aos seus constituintes e à sua ultra-estrutura organizacional. Devido a este comportamento viscoelástico, esta tende a não retornar ao seu estado inicial de maneira imediata, quando submetida a uma deformação temporária em sua estrutura por meio da aplicação de forças externas (Silver et al., 2001; Silver et al., 2003). Esse fenômeno ocorre na pele jovem e é acentuado na pele envelhecida (Oba et al., 2002).

Assim, as características mecânicas da pele resultam da interação entre todos os seus componentes quando a força externa é aplicada e, dependendo do sentido e da intensidade dessa força, pode-se deformar a pele em maior ou menor profundidade. A derme, em geral, é a camada dominante em relação ao comportamento mecânico da pele em condições normais (Silver et al., 2003). Entretanto, 
existe certa controvérsia na literatura, sobre a interação entre o grau de umectação cutânea e a sua elasticidade. Existem autores (Dobrev, 2000; Jemec, Serup, 1990) que defendem que o aumento do conteúdo aquoso do estrato córneo aumenta a possibilidade de distensão da pele, e autores contrários a esta correlação. É possível que esta correlação exista quando, empregando-se métodos baseados nas propriedades visco-elásticas da pele, a profundidade da medição realizada é pequena, restringindo-se apenas à superfície da pele. Nesse caso, a medida obtida poderia depender tanto das propriedades da pele em si, quanto da intensidade da umectação proporcionada pelo produto cosmético. Já no caso de medidas mais profundas, ou seja, onde a sonda do equipamento atinge também a derme, essa poderia ser considerada como sendo o fator determinante dos resultados obtidos (Dobrev, 2000).

A derme é composta principalmente por colágeno, elastina e glicosaminoglicanos. As principais funções do colágeno são a sustentação e a firmeza da pele, sendo que a alteração em suas fibras terá influência sobre as propriedades viscoelásticas. $\mathrm{O}$ componente elástico deve-se, principalmente, à presença de fibras de elastina, perpendicularmente e paralelamente dispostas em relação à superfície cutânea (derme papilar e reticular, respectivamente) e entrelaçadas entre si; já o componente viscoso, por sua vez, deve-se principalmente às fibras de colágeno, que são grossas, grandes e não ramificadas (Rieger, 2000; Lépori, 2002).

No caso da elasticidade cutânea, o envelhecimento cronológico e o fotoenvelhecimento (causado por fatores externos, principalmente devido à exposição solar) alteram esta propriedade devido à modificações estruturais na derme, devido principalmente às modificações estruturais que ocorrem nas fibras de elastina. A radiação UVA, por exemplo, penetra na pele até a derme, podendo provocar danos nas fibras elásticas e nas fibras de colágeno, causando envelhecimento precoce. A elasticidade também pode ser alterada por formulações que atuem na enzima elastase que, por sua vez, atua sobre as fibras de elastina, sendo esta uma enzima proteolítica do grupo das metaloproteinases (Labat-Robert et al., 2000).

O estilo de vida (hábito de fumar, consumo de álcool ou drogas entorpecentes, má alimentação e qualidade do sono) e o uso de produtos cosméticos e medicamentos também podem alterar as propriedades mecânicas da pele. Além disso, as condições ambientais também exercem ação sobre esta característica (Fukuda, Morimoto, 2001a,b).

A interpretação de alterações nas propriedades mecânicas da pele, frente ao uso de formulações cosméticas, tem representado um desafio para os pesquisadores. Estas alterações têm sido analisadas por diferentes metodologias, que são baseadas em deformação da pele pela aplicação de sucção, ressonância ou torção (Tada, Kanamaru, 2002). Cada um destes métodos possui as suas peculiaridades e, dentre os diversos equipamentos disponíveis, um dos mais empregados atualmente baseia-se na sucção e alongamento da pele, sendo que, um exemplo a ser citado é o equipamento Cutometer ${ }^{\circledR}$ (Campos et al., 2008). Os estudos devem ser sempre criteriosamente realizados, a fim de reduzir ao máximo a interpretação errônea dos resultados obtidos. Algumas das variáveis obtidas por estes equipamentos estão correlacionadas com a espessura da epiderme dos voluntários (Hendriks et al., 2003).

Devido à natureza viscoelástica da pele, existe uma relação entre o componente viscoso (viscoelasticidade) e o componente elástico (elasticidade) da mesma. Assim, quando a ação de determinado produto cosmético é avaliada, esta relação deve ser levada em consideração, visto que, em alguns casos, a comprovação dos efeitos das formulações é realizada de maneira indireta. Portanto, é necessário salientar que várias variáveis, tais como, as relações viscoelasticidade/elasticidade, retração imediata/ distensão total da pele após sucção, devem ser analisadas em conjunto e muito cuidado deve ser tomado para se chegar a uma conclusão quanto ao efeito de determinado produto (Gonçalves, Maia Campos, 2004; Campos et al., 2008).

\section{CONSIDERAÇÕES FINAIS}

A grande vantagem das técnicas de biofísica da pele é a avaliação nas reais condições de uso dos produtos cosméticos. Assim, eles são opções de escolha por parte da comunidade científica do segmento cosmético. Por outro lado, estes métodos apresentam ainda algumas limitações, pois, para observar diferenças nas propriedades mecânicas de camadas mais profundas da pele após o uso de determinado produto, é necessário, muitas vezes, que a utilização desse produto seja diária e por um período prolongado. Além disso, a seleção de voluntários, nestes casos, deve ser baseada não somente na pele fotoenvelhecida e/ou desidratada, mas também na pele madura, onde já se iniciaram os processos de perda do relevo cutâneo, do tônus e ressecamento progressivo, característico do envelhecimento fisiológico, ocasionado por fatores intrínsecos.

Para a interpretação dos resultados obtidos por metodologias não invasivas, é necessário profundo conhecimento da estrutura da pele e de sua fisiologia. Apesar do grande investimento que tem sido realizado no desenvolvimento de novos equipamentos e metodologias para possibilitar a realização de estudos in vivo na pele humana, 
existe ainda a necessidade da utilização de metodologias complementares, pois esses equipamentos ainda não permitem afirmar o mecanismo de ação dos produtos em estudo, apenas mostram se o produto ocasionou ou não alguma modificação nas variáveis avaliadas.

Porém, antes do advento das técnicas de Biofísica e imagem da pele, os métodos não invasivos eram baseados apenas em observações clínicas e avaliação sensorial, apreciações estas de caráter qualitativo, já que, em função de sua subjetividade, quantificar para a realização de uma análise estatística era uma tarefa difícil. Assim, a realização de estudos clínicos considerando as propriedades biomecânicas da pele tem sido um grande avanço na avaliação de produtos dermocosméticos.

Além disso, esses métodos têm maior validade quando realizados em conjunto, ou seja, é importante reunir os resultados oriundos de diferentes variáveis, antes de se chegar a uma conclusão quanto ao efeito do uso de determinado produto; isto tendo-se em vista a complexidade da biologia da pele e dos diversos mecanismos envolvidos na ação de produtos específicos, tais como aqueles com ação antienvelhecimento.

Pelo exposto, pode-se observar que os métodos nãoinvasivos baseados no uso de equipamentos da área de biofísica e imagem da pele são de aplicação recente, principalmente quando utilizados em associação com outras técnicas, objetivas (avaliação instrumental) e subjetivas (sensorial, percepção da eficácia e evidências clínicas).

Cumpre salientar ainda que a compreensão da filosofia destes métodos pode auxiliar no entendimento e na interpretação da avaliação das condições da pele, tanto na pele eudérmica, quanto na pele com algum tipo de alteração, por um outro ângulo, até então não considerado por profissionais da área de saúde. Assim, para a indicação de produtos com finalidades terapêuticas ou cosméticas, a observação clínica realizada pelo dermatologista das melhoras resultantes do produto na pele do paciente têm sido cada vez mais associada a estudos científicos, tais como a avaliação histopatológica da pele em tratamento. Tem sido utilizado ainda, como apelo de vendas pelos fabricantes dos produtos, o emprego de métodos in vitro onde não se utilizam animais de experimentação e nem técnicas invasivas.

A realização de estudos por métodos in vivo é de fundamental importância na elucidação de efeitos de formulações tópicas, pois variáveis que interferem na eficácia de substâncias ativas, como sua penetração cutânea e metabolização, não podem ser previstas por métodos alternativos realizados in vitro e para as formulações atuarem na derme, suas substâncias ativas precisam inicialmente penetrar na epiderme viável e depois na derme. Além disso, os métodos in vitro não podem ser correlacionados às reais condições de uso dos produtos, em função da variabilidade existente entre indivíduos de uma mesma espécie.

Quando se considera o complexo mecanismo envolvido no envelhecimento cutâneo, para afirmar-se que um produto tem ação antienvelhecimento, julgamos necessária a avaliação da pele como um todo, ou seja, o estudo das alterações que podem ocorrer na epiderme, tais como mudanças nas condições de hidratação, na velocidade de renovação celular, no padrão de pigmentação, na perda de água transepidérmica, etc., e na derme (firmeza, sustentação e elasticidade) (Nangia et al., 1998). Essas alterações se traduzem clinicamente por uma pele aparentemente mais ou menos hidratada, lisa e, ainda, com mais ou menos rugas. Em relação ao uso de produtos cosméticos com a finalidade de prevenir e retroceder o envelhecimento cutâneo, os que prometem melhorar as características viscoelásticas da pele têm tido grande destaque e aceitação pelo consumidor, e vários produtos têm sido lançados com este apelo de vendas.

Concluindo, a utilização das diversas técnicas de biofísica e imagem da pele representa um avanço na comprovação dos efeitos de produtos dermocosméticos e na elucidação de tais efeitos sobre a pele nas condições reais de uso. Tais resultados são obtidos por meio de protocolos experimentais devidamente elaborados. Finalizando, esses métodos vêm sendo cada vez mais aprimorados do ponto de vista tecnológico e mais utilizados em estudos de eficácia de produtos dermocosméticos.

\section{AGRADECIMENTOS}

À Fundação de Amparo à Pesquisa do Estado de São Paulo, FAPESP.

\section{REFERÊNCIAS}

AMBROISINE, L.; EZZEDINE, K.; ELFAKIR, A.; GARDINIER, S.; LATREILLE, J.; MAUGER, E.; TENENHAUS, M.; GUINOT, C. Relationships between visual and tactile features and biophysical parameters in human facial skin. Skin Res. Technol., Malden, v.13, n.2, p.176-183, 2007.

BARAN, R.; MAIBACH, H. Textbook of cosmetic dermatology. New York: Martin Dunitz, 1998. p.245-263.

BAZIN, R.; FANCHON, C. Equivalence of face and volar forearm for the testing of moisturizing and firming effect of cosmetics in hydration and biomechanical studies. Int. J. Cosmet. Sci., Oxford, v.28, n.6, p.453-460, 2006. 
BENAIGES, A.; MARCET, P.; ARMENGOL, R.; BETES, C.; GIRONÉS, E. Study of the refirming effect of a plant complex. Int. J. Cosmet. Sci., Oxford, v.20, n.4, p.223-233, 1998.

BENSON, H. A. E. Transdermal drug delivery: penetration enhancement techniques. Curr. Drug Deliv., Brisbane, v.2, p.23-33, 2005.

BERARDESCA, E. Racial differences in skin function. Acta Derm. Venereol., Oslo, v.185, p.44-46, 1994.

BERARDESCA, E.; BORRONI, G. Instrumental evaluation of cutaneous hydration, Clin. Dermatol., Philadelphia, v.13, n.4, p.323-327, 1995.

BERARDESCA, E.; MAIBACH, H. I. Noninvasive Bioengineering Assessment of Psoriasis. Int. J. Dermatol., Rochester, v.28, n.3, p.157-160, 2007.

BRASIL - Resolução n ${ }^{\circ}$ 211, de 14/07/2005 - Agência Nacional de Vigilância Sanitária. Disponível em: <www.anvisa.gov.br>. Acesso em: 10 Set. 2008.

CAMPOS, P. M. B. G. M.; GONÇALVES, G. M. S.; GASPAR, L. R. In vitro antioxidant activity and in vivo efficacy of topical formulations containing vitamin $\mathrm{C}$ and its derivatives studied by non-invasive methods. Skin Res. Technol., Malden, v.14, p.376-380, 2008.

CHILCOTT, R. P.; FARRAR, R. Biophysical measurements of human forearm skin in vivo: effects of site, gender, chirality and time. Skin Res. Technol., Malden, v.6, n.2, p.64-69, 2008.

DIKES, P. J. What are meters measuring? Int. J. Cosmet Sci., Oxford, v.24, p.241-245, 2002.

DIRIDOLLOU, S.; BERSON, M.; VABRE, V.; BLACK, D.; KARLSSON, B.; AURIOL, F.; GREGOIRE, J. M.; YVON, C.; VAILLANT, L.; GALL, Y.; PATAT, F. An in vivo method for measuring the mechanical properties of the skin using ultrasound. Ultrasound Med. Biol., Cincinnati, v.24, n.2, p.215-224, 1998.

DOBREV, H. Use of cutometer to assess epidermal hydration. Skin Res. Technol., Malden, v.6, n.4, p.239-244, 2000.
DUJARDIN, N., STAES, E., KALIA, Y.; CLARYS, P.; GUY, R.; PREAT, V. In vivo assessment of skin electroporation using square wave pulses. J. Control. Release, Amsterdam, v.79, n.1-3, p.219-227, 2002.

EDWARDS, C.; MARKS, R. Evaluation of biomechanical properties of human skin Clin. Dermatol., Philadelphia, v.13, n.4, p.375-389, 1995.

EGAWA, M.; OGURI, M.; HIRAO, T.; TAKAHASHI, M.; MIYAKAWA, M. The evaluation of skin friction using a frictional feel analyzer. Skin Res. Technol., Malden, v.8, n.1, p.41-51, 2002.

ES-SPIEKMAN, W; LUCASSEN, G.W. Skin characterization: Human skin water content versus lipid content measured by corneometer, sebumeter and ATR-FTIR spectroscopy. In: IFSCC CONGRESS - Cosmetic Science for the New Century, 21., 2000, Berlin. Anais. Berlin: 2000. 1 CDROM.

FTHENAKIS, C. G.; MAES, D. H.; SMITH, W. P. In vivo assessment of skin elasticity using ballistometry. J. Soc. Cosmet. Chem., New York, v.42, n.4, p.211-222, 1991.

FUKUDA, S.; MORIMOTO, K. Lifestyle, stress and cortisol response: Review I. Environ. Health. Prev. Med., Osaka, v.6, n.1, p.9-14, 2001a.

FUKUDA, S.; MORIMOTO, K. Lifestyle, stress and cortisol response: Review II. Environ. Health. Prev. Med., Osaka, v.6, n.1, p.15-21, 2001 b.

GASPAR, L.R.; GONÇALVES, G. M. S.; PEREIRA, L. H. T. R.; MAIA CAMPOS, P. M. B. G. Aplicação e padronização de metodologias não-invasivas para avaliação de eficácia. Cosm. Toil., São Paulo, v.13, n.5, p.68-73, 2001.

GONÇALVES, G. M. S.; MAIA CAMPOS, P. M. B. G. Use of Cutaneous Bioengineering Techniques to Assess the Effects of Anti-Ageing Cosmetic Products. In: IFSCC CONGRESS, 25., 2004, Orlando. Anais. Orlando: 2004. 1 CD-ROM.

GUIA de Estabilidade de Produtos Cosméticos - Séries Temáticas, volume 1, maio de 2004. Disponível em: <www. anvisa.gov.br>. Acesso em: 5 Mar. 2006.

HADGRAFT, J. Modulation of the barrier function of the skin. Skin Pharmacol. Appl. Skin Physiol., Berlin, v.14, supl.1, n.1, p.72-81, 2001. 
HAFTEK, M. The stratum corneum. Ann. Dermatol. Venereol., Issy les Moulineaux, v.129, n.1, p.117-122, 2002.

HASHIMOTO-KUMASAKA, K.; TAKAHASHI, K.; TAGAMI, H. Electrical measurement of the water content of the stratum corneum in vivo and in vitro under various conditions: comparison between skin surface Hygrometer and Corneometer in evaluation of the skin surface hydration state. Acta Derm. Venereol. Suppl., Oslo, v.73, n.5, p.335339, 1993.

HENRY, F.; VAN LOOK, R.; GOFFIN, V.; FISSETTE, J.; PIERARD, G. E. Mechanical properties of skin and liposuction. Dermatol. Surg., Malden, v.22, n.6, p.566-568, 1996.

JEMEC, G. B. E.; SERUP, J. Epidermal hydration and skin mechanisms: the relationship between electrical capacitance and the mechanical properties of human skin in vivo. Acta Derm. Venereol. Suppl., Oslo, v.70, n.3, p.245-247, 1990.

KALIA, Y. N.; NONATO, L. B.; LUND, C. H.; GUY, R. H. Development of Skin Barrier Function in Premature Infants. J. Invest. Dermatol., Chapel Hill, v.111, n.2, p.320-326, 1998.

KEMENADE, P. M.; HOUBEN, M. M. J.; HUYGHE, J. M. Do osmotic forces play a role in the uptake of water by human skin? Skin Res. Technol., Malden, v.10, n.2, p.109$112,2004$.

LABAT-ROBERT, J.; FOURTANIER, A.; BOYERLAFARGUE, B.; ROBERT, L. Age dependent increase of elastase type protease activity in mouse skin Effect of UVirradiation. J. Photochem. Photobiol. B, Biol., Lausanne, v.57, n.2-3, p.113-118, 2000.

LE FUR, I. Facial Skin Circadian Rhythms of Healthy Women Investigated Using Non-Invasive Methods. In: IFSCC CONGRESS, 22., Edinburgh, 2002. Anais. Edinburgh: 2002. 1 CD-ROM.

LEOW, Y.; MAIBACH, H.I. Cigarette smoking, cutaneous vasculature, and tissue oxygen. Clinics in Dermatology, Philadelphia, v.16, n.5, p.579-584, 1998.

LÉPORI, L. R. Miniatlas: a pele. São Paulo: Soriak Comércio e Promoções S.A., 2002. 204p.
LODÉN, M. Biophysical methods of providing objective documentation of the effects of moisturizing creams Skin Res. Technol., Malden, v.1, n.3, p.101-108, 2006.

LODÉN, M. Skin barrier function: effects of moisturizers. Cosmetics \& Toiletries, Carol Stream, v.116, n.6, p.31-38, 2001.

LOMUTO, M.; PELLICANO, R.; GIULIANI, M. Equipment available for bioengineering of the skin. Clin. Dermatol., Philadelphia, v.13, n.4, p.409-415, 1995.

MADISON, K.C. Barrier Function of the Skin: "La Raison d'Être" of the Epidermis J. Invest. Dermatol., Chapel Hill, v.121, n.2, p.231-241, 2003.

MAES, D.; MARENUS, K.; SMITH, W. P. Invisible irritation: a new look at product safety. Cosm. Toil., Carol Stream, v.105, p.43-49, 1990.

MILAN, A. L. K.; MILÃO, D.; SOUTO, A. A.; CORTE, T. W. F. Estudo da hidratação da pele por emulsões cosméticas para xerose e sua estabilidade por reologia. Rev. Bras. Cienc. Farm., São Paulo, v.43, n.4, p.649-657, 2007.

NANGIA, A.; PATIL, S.; BERNER, B.; BOMAN, A.; MAIBACH, $\mathrm{H}$. In vitro measurement of transepidermal water loss: a rapid alternative to tritiated water permeation for assessing skin barrier functions. Int. J. Pharm., Amsterdam, v.170, n.1, p.33-40, 1998.

OBA, A.; GOMI, T.; NISHIMORI, Y.; GRAVES, C.; PEARSE, A.; EDWARDS, C. A non-invasive method for measuringinvisible subcutaneous uv damage. In: IFSCC CONGRESS, 22. - 2002, Edinburgh. Anais. Edinburgh: 2002. 1 CD-ROM.

PETERSEN, R. D. Ceramides: key components for skin protection. Cosm. Toil., Carol Stream, v.107, n.2, p.45-49, 1992.

PIERARD, G.E.; NIKKELS-TASSOUDJI, N. PIERARDFRANCHMONT, C. Influence of the test area on the mechanical properties of skin. Dermatology (Basel), v.191, n.1, p.9-15, 1995.

PINTO, P.; GALEGO, N.; SILVA, N. Definição de critérios da avaliação dos efeitos sobre a superfície cutânea de cremes hidratantes. I - Análise após uma única aplicação. Rev. Port. Farm., Porto, v.47, n.1, p.23-34, 1997. 
REUTHER, T.; BAYRHAMMER, J.; KERSCHER, M. Use of biophysical techniques to evaluate the physiologic effects of injected hyaluronic acid. Hautarzt, Berlin, v.58, n.12, p.1046-1050, 2007.

RIEGER, M. M. Keratinocyte function - and skin health. Cosm. Toil., Carol Stream, v.107, n.7, p.35-43, 1992.

RIEGER, M. M. Skin. Harry's Cosmeticology. 8.ed. New York: Chemical Publishing Co., 2000. p. 3-38.

RODRIGUES, L. Biofísica da pele: novas perspectivas sobre a fisiologia da pele. Cosm.Toil., São Paulo, v.8, p.51-55, 1997.

ROGIERS, V.; BALLS, M.; BASKETTER, D.; BERARDESCA, E.; EDWARDS, C.; EISNER, P.; ENNEN, J.; LEVEQUE, J. L.; LODEN, M.; MASSON, P.; PARRA, J.; PAYE, M.; PIERARD, G.; RODRIGUES, L.; SCHAEFER, H.; SALTER, D.; ZUANG, V. The potential use of noninvasive methods in the safety assessment of cosmetic products - The report and recommendations of ECVAM/ EEMCO Workshop 36. Altern Lab Anim, Baltimore, v.27, p.515-537, 1999.

ROHR, M.; SCHRADER, K. Climatic influence on cosmetic skin parameters. Curr. Probl. Dermatol., Basel, v.26, p.151164, 1998.

ROSSI, A. B. R.; VERGNANINI, A. L. Mecanismos de hidratação da pele. Cosm. Toil., São Paulo, v.9, n.6, p.3339, 1997.

SCHUPLEIN, R. J.; BRONAUGH, R. L. Percutaneous absortion. In: GOLDSMITH, L.A. (Ed.) Biochemistry and physiology of the skin. New York: Oxford University Press, 1993. p.1255-1295.

SERUP, J.; NORTHEVED, A. Skin elasticity in localised scleroderma (morphoea). J. Dermatol., Tokyo, v.12, n.1, p.52-62, 1985.

SILVER, F. H.; FREEMAN, J.W.; DEVORE, D. Viscoelastic properties of human skin and processed dermis. Skin Res. Technol., Malden, v.7, n.1, p.18-23, 2001.

SILVER, F. H.; SIPERKO, L. M.; SEEHRA, G. P. Mechanobiology of force transduction in dermal tissue. Skin Res. Technol., Malden, v.9, n.1, p.3-23, 2003.
SMITH, C.W.; REN, X.; EVANS, K.E.; DOOLING, P. J.; BURGESS, A.; WIECHERS, J.; ZAHLAN, N. A novel combined experimental-numerical approach for determination of mechanical properties of skin in vivo. In: IFSCC CONGRESS, 22., Edinburgh, 2002. Anais. Edinburgh: 2002. 1 CD-ROM.

SMITH, W. Stratum corneum barrier integrity controls skin homeostasis. Int. J. Dermatol., Rochester, v.21, n.2, p.99106, 1999.

SPENCER, T.S. Dry skin and skin moisturizers. Clin. Dermatol., Philadelphia, v.6, n.3, p.24-28, 1988.

SUMMERS, R. S.; SUMMERS, B.; CHANDAR, P. ; FEINBERG, C. ; GURSKY, R. ; RAWLINGS, A. V. The effect of lipids, with and without humectant, on skin xerosis. J. Soc. Cosmet. Chem., New York, v.47, n.1, p.2739, 1996.

TABATA, N.; OGOSHI, K.; ZHEN, Y.X.; KLIGMAN, A.M.; H. TAGAMI, H. Biophysical Assessment of Persistent Effects of Moisturizers after Their Daily Applications: Evaluation of Corneotherapy. J. Dermatol., Tokyo, v.200, n.4, p.308313,2000 .

TADA, A.; KANAMARU, A. Restoring collagen fibre bundle structure damage by photoaging vis fibroblast interaction with fibronectin. In: IFSCC CONGRESS, 22., Edinburgh, 2002. Anais. Edinburgh: 2002. 1 CD-ROM.

TSAI, T.F.; MAIBACH, H.I. Water: a possible skin irritant. Cosm.Toil., Carol Stream, v.115, n.2, p.35-37, 2000.

VERDIER-SEVRAIN, S.; BONTÉ, F. Skin hydration: a review on its molecular mechanisms. J. Cosmet. Dermatol., Malden, v.6, n.2, p.75-82, 2007.

WALKER, A.P.; BASKETTER, D.A.; BAVEREL, M.; DIEMBECK, W.; MATTHIES, W.; MOUGIN, D.; PAYE, M.; RÖTHLISBERGER, R.; DUPUIS, J. Test guidelines for assessment of skin compatibility of cosmetic finished products in man. Food Chem. Toxicol., Oxford, v.34, n.7, p.651-660, 1996.

WIECHERS, J.W.; BARLOW, T. Skin moisturization and elasticity originate from at least two different mechanisms. Int. J. Dermatol., Rochester, v.21, p.425-435, 2000. 
YOKOTA, T. , MATSUMOTO, M. , SAKAMAKI, T. , HIKIMA, R. , HAYASHI, S. , YANAGISAWA, M. , KUWAHARA, H., YAMAZAKI, S., OGAWA, T. , HAYASE Classification of sensitive skin and development of a treatment system appropriate for each group. IFSCC Magazine, v.6, n.4, p.303-307, 2003.
ZHAI, H.; BRACHMAN, F.; PELOSI, A.; ANIGBOGU, A.; RAMOS, M. B.; TORRALBA, M. C.; MAIBACH, H. I. A bioengineering study on the efficacy of a skin protectant lotion in preventing SLS-induced dermatitis. Skin Res. Technol., Malden, v.6, n.2, p.77-80, 2008.

Recebido para publicação em 04 de setembro de 2007. Aceito para publicação em 03 de dezembro de 2008. 